\title{
EL EMPRENDIMIENTO COLECTIVO COMO SALIDA LABORAL DE LOS JÓVENES: ANÁLISIS DEL CASO DE LAS EMPRESAS DE TRABAJO ASOCIADO
}

\author{
POR \\ Gustavo LEJARRIAGA PÉREZ DE LAS VACAS ${ }^{1}$, \\ Paloma BEL DURÁN ${ }^{2}$ y \\ Sonia MARTÍN LÓPEZ ${ }^{3}$
}

\section{RESUMEN:}

La falta de oportunidades laborales como trabajadores asalariados para los jóvenes, y las elevadas tasas de desempleo que afectan a la población juvenil, que llegan a duplicar la media europea, han de ser indicadores suficientes para que los jóvenes valoren el emprendimiento como vía de inclusión, o mantenimiento, en el mercado laboral. En este sentido, la vocación emprendedora de los jóvenes españoles se sitúa por debajo de la media de sus homólogos europeos, por lo que se precisa la difusión de las bondades del emprendimiento, tales como la posibilidad de obtener empleos de calidad y estables que se adecúen en mayor medida a la formación obtenida, así como su apoyo.

Pero no sólo ha de valorarse la alternativa de emprender en solitario, sino que también es posible el emprendimiento en colectivo, prestándose en este trabajo especial atención a las empresas de participación, y al papel que pueden desempeñar éstos en las mismas. Estas empresas requieren al menos tres socios para la constitución tanto de una sociedad laboral, como de una sociedad cooperativa de trabajo asociado.

\footnotetext{
${ }^{1}$ Investigador de la Escuela de Estudios Cooperativos de la Universidad Complutense de Madrid. Dirección de correo electrónico: grlejarr@ucm.es

${ }^{2}$ Investigador de la Escuela de Estudios Cooperativos de la Universidad Complutense de Madrid. Dirección de correo electrónico: pbeldura@ucm.es

${ }^{3}$ Investigador de la Escuela de Estudios Cooperativos de la Universidad Complutense de Madrid. Dirección de correo electrónico: smartin@ucm.es
}

REVESCO N 112 - Extraordinario en Homenaje al Profesor Alfonso Carlos MORALES

GUTIÉRREZ - ISSN: 1885-8031 - www.ucm.es/info/revesco

http://dx.doi.org/10.5209/rev_REVE.2013.v112.43068

Fecha de recepción: 31/07/2013

Fecha de aceptación: 24/09/2013 
En este contexto, se estudia la percepción del emprendimiento, y de las empresas de trabajo asociado, entre los jóvenes universitarios de la Universidad Complutense de Madrid, así como las iniciativas que se están llevando a cabo desde esta institución para fomentar, difundir y apoyar estas iniciativas emprendedoras.

Palabras Clave: jóvenes estudiantes, emprendimiento, empresas de trabajo asociado, sociedades laborales, sociedades cooperativas de trabajo asociado

Claves Econlit: L260, J540, M130

\title{
COLLECTIVE ENTREPRENEURSHIP AS A WORK OUT FOR YOUNG PEOPLE: ANALYSIS OF THE CASE OF LABOUR MANAGED FIRMS
}

\begin{abstract}
:
The lack of job opportunities as employee for youth people, and the high rates of unemployment, higher than European average, are indicators that young people have to take account young to value the entrepreneurship as a mean of inclusion, or maintenance in the labor market. In this sense, the entrepreneurial spirit of young Spanish people is below the European average, so it's necessary the diffusion of the benefits of entrepreneurship, such as the possibility of obtaining stable and quality jobs more according to the training received, as well as its support.
\end{abstract}

But not only has to be valued the option to undertake alone, but it is also possible in a collective venture, paying special attention in this work to labour managed firms, and the role they can play in them. These companies require at least three partners for the creation of both, an employee-owned company, as an associated labour cooperative.

In this context, we study the perception of the entrepreneurship, and labour managed firms, among university students from the Universidad Complutense de Madrid, and the initiatives that are being carried out from this institution to promote, disseminate and support these entrepreneurial initiatives.

Keywords: young students, entrepreneurship, labour managed firms, employee-owned companies, associated labour cooperatives 


\section{INTRODUCCIÓN}

Durante los últimos años, como consecuencia de la crisis económica, el problema del desempleo se ha convertido en una de las preocupaciones más importantes de la población, y en especial de los más jóvenes. Desde el año 2008 las cifras de desempleados no han parado de crecer, a diferencia de lo que ocurría en los años anteriores. Así, según datos de la Encuesta de Población Activa (EPA), la cifra total de parados en el año 2012 ascendía a 5.769.000 personas, de las que el 43,83 por ciento tenían menos de 35 años.

Si se considera como población joven a los que se encuentran en edades comprendidas entre los 16 y los 34 años, se observa que es el colectivo de los que tienen entre 25 y 29 años es el más numeroso, seguido de los que tienen entre 30 y 34 años. Un dato positivo es que a partir del año 2010 se ha frenado el importante incremento iniciado en el año 2008. No obstante, salvo en el grupo de los que tienen entre 30 y 34 años, todavía el crecimiento continúa siendo positivo en relación con los datos del año 2011, tal y como aparece recogido en la Tabla 1.

Tabla 1 - Evolución del número de desempleados entre 2007 y 2012

\begin{tabular}{|l|r|r|r|r|r|r|}
\hline \multicolumn{1}{|c|}{ Años } & \multicolumn{1}{|c|}{2007} & \multicolumn{1}{c|}{2008} & \multicolumn{1}{c|}{2009} & \multicolumn{1}{c|}{2010} & 2012 \\
\hline De 16 a 19 & 157,4 & 211,1 & 249,1 & 236,8 & 219,6 & 220,9 \\
\hline $\begin{array}{l}\text { Var. } \\
\text { interanual }\end{array}$ & $0,32 \%$ & $34,12 \%$ & $18,00 \%$ & $-4,94 \%$ & $-7,26 \%$ & $0,59 \%$ \\
\hline De 20 a 24 & 285,3 & 381,9 & 592,4 & 615,2 & 669,4 & 724 \\
\hline $\begin{array}{l}\text { Var. } \\
\text { interanual }\end{array}$ & $-0,42 \%$ & $33,86 \%$ & $55,12 \%$ & $3,85 \%$ & $8,81 \%$ & $8,16 \%$ \\
\hline De 25 a 29 & 287,4 & 422,8 & 661,9 & 718,6 & 742,5 & 822,5 \\
\hline $\begin{array}{l}\text { Var. } \\
\text { interanual }\end{array}$ & $-11,13 \%$ & $47,11 \%$ & $56,55 \%$ & $8,57 \%$ & $3,33 \%$ & $10,77 \%$ \\
\hline De 30 a 34 & 260,3 & 377,8 & 628,1 & 703,2 & 845,4 & 761,1 \\
\hline $\begin{array}{l}\text { Var. } \\
\text { interanual }\end{array}$ & $1.833,80$ & $2.590,60$ & $4.149,50$ & $4.632,40$ & 4999 & 5769 \\
\hline Total & $-0,18 \%$ & $41,27 \%$ & $60,18 \%$ & $11,64 \%$ & $7,91 \%$ & $15,40 \%$ \\
\hline $\begin{array}{l}\text { Var. } \\
\text { interanual }\end{array}$ & $45,14 \%$ & $66,25 \%$ & $11,96 \%$ & $20,22 \%$ & $-97 \%$ \\
\hline
\end{tabular}

REVESCO No 112 - Extraordinario en Homenaje al Profesor Alfonso Carlos MORALES

GUTIÉRREZ - ISSN: 1885-8031 - www.ucm.es/info/revesco 
Fuente: Elaboración propia a partir de datos de la Encuesta de Población Activa (EPA) elaborada por el INE. Disponible en Internet en: http://www.ine.es/jaxiBD/menu.do? $L=0 \&$ divi $=E P A \&$ his $=3 \&$ type $=d b$ Nota: Datos en miles de personas. Fecha de consulta: 01 julio de 2013.

En línea con lo expuesto anteriormente, la tasa de paro de la población española se ha situado en el año 2012 en el 25,03 (más del doble de la tasa del 9,16 del año 2005). Sin embargo, dicha tasa media es superada ampliamente en el caso de la población más joven. Así, en los jóvenes entre 16 y 19 años se sitúa en el 72,65 por ciento, entre los jóvenes entre 20 y 24 años, en el 49,13 por ciento, entre los jóvenes entre 25 y 29 años en el 32,19 por ciento, y entre los jóvenes entre 30 y 34 años en el 25,29 por ciento.

Ante este escenario, dadas las dificultades de encontrar trabajo por cuenta ajena, el emprendimiento se valora positivamente como una oportunidad, bien para incorporarse, mantenerse (para aquellos que debido a la inestabilidad laboral consideren que pueden perder su puesto de trabajo en un futuro próximo), o en el caso de los desempleados, para reincorporarse, al mercado laboral. En concreto, la opción del emprendimiento colectivo como empresas de trabajo asociado (sociedades laborales y sociedades cooperativas de trabajo asociado) es una alternativa válida para salir del desempleo y reinsertarse en el mercado laboral, contribuyendo así a dinamizar la economía, como han demostrado en recesiones previas (GRÁVALOS y POMARES: 2001; BEN-NER: 1998; TOMÁS: 1995). Por otra parte, en los epígrafes siguientes también se analizará la percepción que tienen los jóvenes estudiantes universitarios del emprendimiento, y de las empresas de trabajo asociado, centrándonos para ello en una muestra de estudiantes de la Universidad Complutense de Madrid. Por último, se explicarán distintas medidas que se están llevando a cabo para el fomento del emprendimiento en el ámbito universitario, y en concreto en la Universidad Complutense de Madrid.

\section{EL PAPEL DE LAS EMPRESAS DE TRABAJO ASOCIADO COMO FÓRMULAS DE EMPRENDIMIENTO COLECTIVO.}

Las empresas de trabajo asociado (sociedades cooperativas de trabajo asociado, y sociedades laborales, anónimas y de responsabilidad limitada) son empresas de participación caracterizadas por ser organizaciones en las lo que les confiere poder de decisión para establecer los objetivos de la empresa - es decir, para tener la condición de socio - es 
precisamente la participación, como protagonistas, promotores, actores, en el proceso de producción-distribución de bienes y servicios, que permiten pasar de su condición de factores a la de productos, respectivamente, como proveedores de unos y/o como consumidores de los otros. En estas empresas las personas y sus intereses en el proceso de producción y distribución se superponen al capital (GARCÍA-GUTIÉRREZ: 2002, p. 108). Así las cosas, mientras que los socios trabajadores participan tanto en los flujos productivo o reales, como en los informativo-decisiones, y en los económico-financieros, en el caso de los socios colaboradores y capitalistas, según se trate de una sociedad cooperativa de trabajo asociado o de una sociedad laboral, su participación queda restringida a los flujos informativodecisionales y a los flujos financieros.

Desde la Unión Europea se ha tratado de promover y favorecer en los estados miembros la implantación de planes de participación dirigidos a los trabajadores en las empresas en las que trabajan, con independencia de su tamaño empresarial. La mayor implicación de los trabajadores en la empresa tiene su reflejo en la mejora de la productividad, de la competitividad, de la calidad laboral y de la rentabilidad en la empresa. Así, se permite la creación de vínculos más estables entre el trabajador y la empresa, se reduce la rotación, se incrementa la estabilidad laboral y aumenta la motivación.

La participación de los trabajadores en las empresas en las que trabajan puede materializarse vía participación en los beneficios (los trabajadores participan de forma directa en los resultados empresariales al estar parte de su retribución salarial vinculada al resultado positivo de la empresa), o vía participación en el capital social de la empresa (la participación del trabajador es indirecta, mediante el cobro de dividendos o revalorización de los títulos).

Desde la aparición de las primeras recomendaciones, y la publicación del primer informe PEPPER ("Promotion of Employee Participation in Profits and Enterprise Results", "Fomento de la participación de los trabajadores en los beneficios y los resultados de la empresa") en el año 1991, se han realizado avances en la difusión de la participación financiera de los trabajadores, tal y como confirma el IV Informe PEPPER del año 2009 sobre evaluación y comparación de la participación financiera de los trabajadores en la Unión Europea de los 27. No obstante, los planes de participación financiera no están los suficientemente extendidos entre los estados miembros, dado que existen algunos obstáculos como las diferencias culturales, los distintos sistemas fiscales, jurídicos, regímenes de 
cotización a la Seguridad Social, y costes de implantación, entre otros, que hacen que su difusión se vea ralentizada. Pero a pesar de ello desde la Unión Europea se considera que se trata de un asunto en el que han de seguir trabajando todos los estados miembros. Así, en el año 2010, el Comité Económico y Social Europeo elaboró el Dictamen SOC/371 sobre participación financiera de los trabajadores en Europa, con el que se pretende animar a Europa a elaborar un marco de referencia que fomente la cohesión social y económica, agilizando la aplicación de la participación financiera de los trabajadores en los distintos niveles. De cara al futuro, la participación financiera de los trabajadores puede ayudar a las PYMEs a mejorar su nivel de competitividad contribuyendo a garantizar un futuro sostenible, a incrementar el poder adquisitivo de la región al revertir parte de los resultados financieros en los trabajadores, a combatir el cambio demográfico, así como a favorecer la transmisión de las empresas.

Entre los modelos propuestos por la Unión Europea, dentro del ámbito de los informes PEPPER, a seguir para la difusión de la participación financiera entre los trabajadores, en el caso de España, se encuentran la sociedad cooperativa de trabajo asociado, y la sociedad laboral. Estas fórmulas jurídicas permiten la concreción de la participación financiera de los trabajadores que trabajan en pequeñas y medianas empresas, o en microempresas. La participación financiera de los socios trabajadores en las sociedades cooperativas de trabajo asociado está vinculada a la participación en los resultados en función de la participación del socio trabajador en la actividad cooperativizada, mientras que en el caso de las sociedades laborales la participación financiera se concreta tanto en la participación en la propiedad en proporción a su participación en el capital social y por tanto, en el proceso de toma de decisiones de la sociedad, como con la participación en los resultados en función de la aportación realizada al capital social (MARTÍN, LEJARRIAGA, ITURRIOZ: 2007).

Cabe señalar que en ambos tipos de sociedades la legislación establece que el número mínimo de socios para poderlas constituir ha de ser tres, dado que ningún socio puede poseer más de un tercio del capital social, por lo que se trata de fórmulas jurídicas de emprendimiento colectivo. De ellos al menos dos socios han de ser socios trabajadores, pudiendo ser un tercero socio colaborador o socio capitalista. No obstante, varía el porcentaje de participación de los socios trabajadores. Así, en el caso de las sociedades cooperativas de trabajo asociado la participación mínima conjunta de todos los socios trabajadores es del 55 por ciento, frente al porcentaje que han de poseer los socios trabajadores de las sociedades 
laborales del 51 por ciento ${ }^{4}$. Por tanto, en ambos tipos de sociedades son los socios trabajadores los que en cualquier caso tienen el control de la empresa, al poseer la mayoría del capital social.

Dicha limitación legal del número mínimo de socios que han de constituir este tipo de empresas ha sido una de las principales causas de descalificación de diversas empresas de trabajo asociado que han continuado desarrollando su actividad empresarial transformándose en otras formas jurídicas, aun conservando su carácter participativo, como consecuencia del abandono de algún socio trabajador al no poder ser reemplazado (LEJARRIAGA y MARTÍN: 2010; p.378).

Las empresas de trabajo asociado se caracterizan, además de porque sus promotores desempeñan el doble papel de socios y trabajadores, por basarse en una serie de valores que van más allá de los puramente económicos, teniendo también en cuenta los de índole social, lo que contribuye a la creación de puestos de trabajo de calidad y estables (MONTOLIO: 2002; p. 11). De hecho, a lo largo de su historia han desempeñado un importante papel en términos de creación y mantenimiento de puestos de trabajo (DICKTEIN: 1991), tanto para sus socios como para terceros no socios. Además, han demostrado que se adaptan en mejor medida que otro tipo de empresas a las circunstancias cambiantes del entorno, debido a la mayor implicación de sus socios trabajadores, y contribuyen al desarrollo territorial mediante fórmulas democráticas, a una mayor integración y a la cohesión social, aportando al mismo tiempo riqueza, son sensibles a los aspectos relativos a la responsabilidad social, y no se deslocalizan, lo que favorece la creación de riqueza y empleo en las zonas en las que se encuentran (CLEMENTE; DÍAZ y MARCUELLO: 2009; p. 35-69; MORALES, MARTÍN y LEJARRIAGA: 2008; GARCÍA-GUTIÉRREZ y LEJARRIAGA: 2004).

Las empresas de participación han contribuido de forma positiva a la economía en períodos de crisis previos al actual. Así, las sociedades cooperativas de trabajo asociado y las sociedades anónimas laborales, jugaron un papel destacado en la década de los setenta cuando los trabajadores de las empresas, para evitar el cierre de las mismas, pasaron a asumir el doble papel de empresarios y trabajadores, conservando así sus puestos de trabajo. Además, han

\footnotetext{
${ }^{4}$ ESPAÑA. LEY 4/1997, de 24 de marzo, de Sociedades Laborales. Art. 5. Dicho límite podrá ser superado cuando se trate de sociedades laborales participadas por el Estado, las Comunidades Autónomas, las Entidades locales o las sociedades públicas participadas por cualquiera de tales instituciones, pero sin alcanzar el límite del 50 por ciento del capital social.
}

REVESCO No 112 - Extraordinario en Homenaje al Profesor Alfonso Carlos MORALES

GUTIÉRREZ - ISSN: 1885-8031 - www.ucm.es/info/revesco 
demostrado presentar mayor flexibilidad y capacidad de adaptación ante circunstancias adversas (MONZÓN: 2010a; p. 361), así como un comportamiento mejor que otras formas jurídicas (MELGAREJO; ARCELUS, y SIMÓN: 2007). Llegándose a la conclusión en determinados estudios "del carácter contra cíclico y reactivo de la economía social en la sociedad occidental" (TOMAS; 1995: p. 25), y de las sociedades laborales en particular ${ }^{5}$ (GRÁVALOS y POMARES: 2001). No obstante, la creación de este tipo de empresas no sólo está ligada exclusivamente a las situaciones de crisis económica, cuando surgen con mayor fuerza que en circunstancias normales, mientras muchas de las empresas capitalistas convencionales tienden a desaparecer, sino que al igual que éstas últimas, también se crean en períodos de crecimiento económico.

Las sociedades de trabajo asociado a lo largo de su historia han contribuido de forma significativa a la generación de riqueza, así como a la creación de empleo de calidad y estable, aunque durante los últimos años haya tenido lugar un cierto estancamiento. Del análisis de la creación de nuevas empresas de participación de trabajo asociado cabe destacar el notable descenso desde el año 2002, habiendo sido el año 2008 el momento en el que tuvo lugar la recesión más importante (reducción del 31,65 por ciento con relación al año anterior, y del 72,97 por ciento si se compara con las 7.700 sociedades creadas en el año 2002). No obstante, hasta el año 2005 la cifra de empresas de participación de trabajo asociado continuó creciendo, al superar el número de nuevas empresas creadas el de las que se daban de baja. En el caso de las sociedades cooperativas de trabajo asociado, tras la importante caída sufrida en el año 2008, en el año 2009 el número de sociedades registradas se incrementó en un 14,69 por ciento, prologándose la tendencia de crecimiento positivo durante el año 2010, aunque a una tasa menor (6,40 por ciento), para volver a reducirse en un 9,31 en el año 2011, e incrementarse un 15,80 por ciento en el año 2012. Sin embargo, las sociedades laborales registradas continuaron disminuyendo en el año 2009, y sólo en el año 2010 presentan un leve crecimiento del 2,20 por ciento. No obstante, durante los años 2011 y 2012 continuaron reduciéndose el número de sociedades laborales registradas. Esto hace que la cifra de empresas de participación de trabajo asociado se sitúe en el año 2012 en 1.739 sociedades, habiendo tenido lugar una reducción del 2,19 por ciento respecto a los datos del año anterior.

\footnotetext{
${ }^{5}$ En este estudio se concluye un comportamiento anticíclico de las Sociedades Laborales en todas las regiones españolas, aunque admitiendo ciertas especificidades regionales.
} 
Tabla 2 - Evolución del número de sociedades laborales (SL) y sociedades cooperativas de trabajo asociado (SCTA) nuevas, registradas en el período 2002-2012

\begin{tabular}{|c|c|c|c|c|c|c|c|c|c|c|c|}
\hline & 2002 & 2003 & 2004 & 2005 & 2006 & 2007 & 2008 & 2009 & 2010 & 2011 & 2012 \\
\hline$S L$ & 6.013 & 5.353 & 4.249 & 3.466 & 2.526 & 2.341 & 1.514 & 1.225 & 1.252 & 1.145 & 1.006 \\
\hline Var. Relativa & $10,25 \%$ & $-10,98 \%$ & $-20,62 \%$ & $-18,43 \%$ & $-27,12 \%$ & $-7,32 \%$ & $-35,33 \%$ & $-19,09 \%$ & $2,20 \%$ & $-8,55 \%$ & $-12,14 \%$ \\
\hline SCTA & 1.703 & 1.500 & 1.353 & 1.020 & 761 & 711 & 572 & 656 & 698 & 633 & 733 \\
\hline Var. Relativa & $-11,58 \%$ & $-11,92 \%$ & $-9,80 \%$ & $-24,61 \%$ & $-25,39 \%$ & $-6,57 \%$ & $-19,55 \%$ & $14,69 \%$ & $6,40 \%$ & $-9,31 \%$ & $15,80 \%$ \\
\hline TOTAL & 7.716 & 6.853 & 5.602 & 4.486 & 3.287 & 3.052 & 2.086 & 1.881 & 1.950 & 1.778 & 1.739 \\
\hline Var. Relativa & $4,55 \%$ & $-11,18 \%$ & $-18,25 \%$ & $-19,92 \%$ & $-26,73 \%$ & $-7,15 \%$ & $-31,65 \%$ & $-9,83 \%$ & $3,67 \%$ & $-8,82 \%$ & $-2,19 \%$ \\
\hline
\end{tabular}

Fuente: Elaboración propia a partir de datos del Ministerio de Empleo y Seguridad Social. Disponible en Internet en: http://www.empleo.gob.es/es/sec_trabajo/autonomos/economia-

soc/EconomiaSocial/estadisticas/index.htm. Fecha de consulta: 01 julio 2013.

En términos de sociedades en situación de alta en la Seguridad Social a finales de 2012 había 18.843 sociedades, de las que 12.294 eran sociedades laborales, y 6.549 sociedades cooperativas de trabajo asociado, lo que supone una reducción en relación a las 19.345 sociedades existentes en el año 2011 del 2,60 por ciento, y del 45 por ciento si se toman como referencia las 34.223 sociedades en situación de alta en la Seguridad Social del año 2005, año en el que se alcanzó la cifra más alta. Por lo que, las reducciones en términos relativos han sido más significativas en términos del número de empresas creadas, que de las que se encuentran en situación de alta en la Seguridad Social.

En lo relativo a los socios trabajadores de las nuevas sociedades de trabajo asociado la evolución ha sido similar a la del número de empresas, aunque en este caso las reducciones en términos anuales de los últimos años han sido ligeramente menores. Durante el año 2011 la reducción del número de socios trabajadores ha sido del 7,48 por ciento, superior al 1,67 por ciento del año anterior, situándose la cifra total de socios trabajadores de las nuevas sociedades de trabajo asociado registradas en el año 2011 en 5.602. En el año 2012 ha habido un importante incremento del número de socios trabajadores de las sociedades cooperativas de trabajo asociado, situándose en 8.436. Sin embargo el número de socios trabajadores de las sociedades laborales todavía continúa reduciéndose aunque a tasas inferiores que en los últimos años. 
Tabla 3 - Evolución del número de socios trabajadores de las nuevas sociedades laborales (SL) y sociedades cooperativas de trabajo asociado (SCTA) registrados en el período 2002-2012

\begin{tabular}{|c|c|c|c|c|c|c|c|c|c|c|c|}
\hline & 2002 & 2003 & 2004 & 2005 & 2006 & 2007 & 2008 & 2009 & 2010 & 2011 & 2012 \\
\hline SL & 14.983 & 13.770 & 11.281 & 8.982 & 6.490 & 5.987 & 4.164 & 3.526 & 3.382 & 3.293 & 3.101 \\
\hline Var. Relativa & $9,73 \%$ & $-8,10 \%$ & $-18,08 \%$ & $-20,38 \%$ & $-27,74 \%$ & $-7,75 \%$ & $-30,45 \%$ & $-15,32 \%$ & $-4,08 \%$ & $-2,63 \%$ & $-5,83 \%$ \\
\hline SCTA & 6.631 & 5.727 & 5.211 & 4.648 & 3.014 & 2.891 & 2.463 & 2.632 & 2.673 & 2.309 & 8.436 \\
\hline Var. Relativa & $-14,88 \%$ & $-13,63 \%$ & $-9,01 \%$ & $-10,80 \%$ & $-35,15 \%$ & $-4,08 \%$ & $-14,80 \%$ & $6,86 \%$ & $1,56 \%$ & $-13,62 \%$ & $265,35 \%$ \\
\hline TOTAL & 21.614 & 19.497 & 16.492 & 13.630 & 9.504 & 8.878 & 6.627 & 6.158 & 6.055 & 5.602 & 11.537 \\
\hline Var. Relativa & $0,79 \%$ & $-9,79 \%$ & $-15,41 \%$ & $-17,35 \%$ & $-30,27 \%$ & $-6,59 \%$ & $-25,35 \%$ & $-7,08 \%$ & $-1,67 \%$ & $-7,48 \%$ & $105,94 \%$ \\
\hline
\end{tabular}

Fuente: Elaboración propia a partir de datos del Ministerio de Empleo y Seguridad Social. Disponible en Internet en: http://www.empleo.gob.es/es/sec trabajo/autonomos/economiasoc/EconomiaSocial/estadisticas/index.htm. Fecha de consulta: 01 de julio 2013.

Según lo expuesto previamente el número de socios trabajadores por nueva sociedad de trabajo asociado en el año 2011 es de 3,15 (siendo de 2,87 en las sociedades laborales, y de 3,65 en las sociedades cooperativas de trabajo asociado).

En cuanto al número de trabajadores dados de alta en la Seguridad Social se han pasado de tasas de variación interanuales de crecimiento de empleo por encima del 5 por ciento (5,46 por ciento en el año 2000 con relación a 1999), a tasas negativas del 10,5 por ciento en 2008 (con relación a 2007). El mayor número de empleos se logró en 2006, con 312.145 trabajadores. Se aprecia un comportamiento similar al de la evolución en el número de empresas. Especialmente alarmante resulta la reducción del empleo en las sociedades laborales limitadas que alcanza una variación negativa en 2008 de más del 20 por ciento. En el año 2010 el número de trabajadores dados de alta en la Seguridad Social en las empresas de trabajo asociado asciende a 149.187, de los que 67.445 pertenecen a sociedades laborales, y 81.742, a sociedades cooperativas de trabajo asociado. Por lo que en el año 2012 ha tenido lugar una reducción del 2,56 por ciento en relación con el año anterior.

Los trabajadores de las empresas de trabajo asociado se caracterizan por ser mayoritariamente varones (62,0 por ciento en las sociedades laborales de responsabilidad limitada, y 46,8 por ciento en las sociedad cooperativas de trabajo asociado), aunque progresivamente se va incrementando la presencia de las mujeres, con edad comprendida entre los 25 y los 39 años (62,8 por ciento en las sociedades laborales de responsabilidad limitada, y 42,1 por ciento en las sociedad cooperativas de trabajo asociado), con contrato 
indefinido (66,0 por ciento en las sociedades laborales de responsabilidad limitada, y 64,1 por ciento en las sociedad cooperativas de trabajo asociado), a tiempo completo (72,0 por ciento en las sociedades laborales de responsabilidad limitada, y 63,0 por ciento en las sociedad cooperativas de trabajo asociado), y cualificados profesionalmente. Los indicadores expuestos anteriormente permiten confirmar la calidad del empleo generado por las empresas de trabajo asociado, dado el elevado porcentaje de contratos indefinidos y a jornada completa.

Por otra parte, el empleo creado por las sociedades de trabajo asociado además de calidad es estable, como lo ponen de manifiesto los datos relativos a la antigüedad de los trabajadores de estas empresas, y a los indicadores de supervivencia de las mismas. Así, el porcentaje de trabajadores con mayor antigüedad se ha incrementado siendo aproximadamente el 33 por ciento en el año 2011 los que presentan una antigüedad superior a cinco años. En cuanto al porcentaje de empresas de economía social que sobreviven cinco años es aproximadamente de la mitad, ascendiendo el porcentaje de trabajadores respecto al origen que sobreviven a los cinco años aproximadamente del 85 por ciento. Por tanto, en términos de supervivencia un dato positivo es el incremento del número medio de años de vida con respecto al período anterior.

En aras de promover la creación de este tipo de empresas como fórmulas de emprendimiento colectivo, la prestación por desempleo en pago único es una medida a la que pueden acogerse los jóvenes desempleados para obtener el dinero de la prestación contributiva que tienen pendiente de percibir, bien para constituir una sociedad cooperativa de trabajo asociado, o una sociedad laboral, de nueva creación, o para incorporarse a alguna ya existente. De esta forma pueden obtener los recursos financieros necesarios para realizar la aportación al capital social, y/o para subvencionar las cuotas a pagar a la Seguridad Social. Desde su aprobación en el año 1985 esta medida ha contribuido de forma eficaz a la creación de empleo, habiéndose puesto de manifiesto durante los últimos años que los fondos percibidos por los beneficiarios no sólo se utilizan para poner en marcha nuevas iniciativas empresariales, sino que también contribuyen al crecimiento y consolidación de las ya existentes. No obstante, las modificaciones legales que han tenido lugar, mejorando en los últimos años las condiciones de acceso a esta medida por parte de los trabajadores autónomos, ha hecho que muchos emprendedores hayan preferido esta opción (FERNÁNDEZ; MARTÍN, y LEJARRIAGA: 2010; p. 105-107).

REVESCO No 112 - Extraordinario en Homenaje al Profesor Alfonso Carlos MORALES

GUTIÉRREZ - ISSN: 1885-8031 - www.ucm.es/info/revesco 
Para concluir, se puede decir que las nuevas sociedades laborales (en concreto las sociedades de responsabilidad limitada laborales) que se crean se ponen en marcha por personas en situación de desempleo que quieren reincorporarse al mercado laboral utilizando para ello la capitalización del desempleo en pago único, como vía de obtención de los recursos financieros que necesitan, y creándose así su propio puesto de trabajo (LEJARRIAGA y MARTÍN, 2010: 371). Sin embargo, las sociedades cooperativas de trabajo asociado son la alternativa utilizada por personas, en la mayor parte de los casos, con empleo pero que desean dar una nueva orientación a su trayectoria laboral y crear su propia empresa.

\section{LA PERCEPCIÓN DE LOS JÓVENES EMPRENDEDORES DE LAS EMPRESAS DE PARTICIPACIÓN}

Este apartado procura poner de manifiesto cuál es la percepción de los jóvenes emprendedores sobre la elección de empresas participativas como base jurídica para la puesta en marcha y el desarrollo de sus iniciativas emprendedoras. De este modo, teniendo en cuenta un entorno que parece indicar que los jóvenes tienen una importancia destacada como trabajadores, y por tanto socios, en las empresas de participación, se pretende radiografiar cómo se enfrentan al emprendimiento, más en concreto, a través de dichas fórmulas de participación.

En general, según la información obtenida del Ministerio de Trabajo e Inmigración ${ }^{6}$, se observa un importante peso relativo de los trabajadores de edades comprendidas entre los 25 y 39 años (en las Sociedades de Responsabilidad Limitada Laborales es importante el peso relativo de los jóvenes menores de 25 años) y que más del 70 por ciento de los trabajadores son cualificados profesionalmente, teniendo una calificación laboral de Ingeniero, licenciados $\mathrm{u}$ otras titulaciones técnicas, aunque el porcentaje de licenciados, ingenieros (técnicos o superiores) y diplomados universitarios sólo alcanza el 13 por ciento.

En particular, el estudio que se desarrolla es en el ámbito universitario, a partir de estudio de casos que se han elegido de forma aleatoria de entre las entrevistas y asesorías realizadas entre marzo y junio de 2010 a través de la Oficina Complutense del Emprendedor $\left(\right.$ COMPLUEMPRENDE) ${ }^{7}$ que es la institución que sirve de nexo de unión entre la

\footnotetext{
${ }^{6}$ http://www.mtin.es/es/sec_trabajo/autonomos/economia-soc/EconomiaSocial/estadisticas/index.htm Fecha de consulta: 15 de junio de 2013.

${ }^{7}$ Ver epígrafe El emprendimiento en el ámbito universitario: la experiencia de la Universidad Complutense de

REVESCO No 112 - Extraordinario en Homenaje al Profesor Alfonso Carlos MORALES

GUTIÉRREZ - ISSN: 1885-8031 - www.ucm.es/info/revesco
} 
Universidad y la realidad empresarial al asesorar, guiar y acompañar las iniciativas empresariales de los jóvenes universitarios. Esta información es contrastada con entrevistas a estudiantes universitarios en septiembre y octubre de $2010^{8}$.

Esta información es contrastada por un estudio desarrollado en el Año Académico 2012-2013 en el que se ha distribuido un cuestionario a estudiantes universitarios en los estudios de la Licenciatura de Administración y Dirección de Empresas de la Universidad Complutense de Madrid y el Grado en Administración y Dirección de Empresas. En concreto se les ha pasado el cuestionario a los alumnos de aquellas asignaturas en las que se estimula el espíritu emprendedor entre los estudiantes.

El análisis se centra en el estudio de la elección de fórmulas jurídicas participativas cuando el colectivo universitario se plantea emprender una actividad. Dichas fórmulas jurídicas son las sociedades cooperativas de trabajo asociado, las sociedades laborales y el trabajo autónomo. Por otro lado, se valora el grado de formación en estas formas jurídicas así como la demanda de información por parte de los emprendedores.

Del análisis de las experiencias tuteladas, acompañadas o asesoradas por la oficina COMPLUEMPRENDE, se desprende una primera aproximación al estado de la cuestión, a saber, cuándo los nuevos emprendedores deciden iniciar una actividad suelen tener una idea preconcebida de la forma jurídica con la que desean iniciar su actividad empresarial. La opción elegida mayoritariamente es la de trabajador autónomo (al considerarse la más adecuada dadas las características de los proyectos que se quieren poner en marcha), y la Sociedad de Responsabilidad Limitada, si bien en algunos casos también muestran interés por la Sociedad Limitada Nueva Empresa.

En primer lugar, cabe preguntarse cuáles son los motivos por los cuales los jóvenes deciden emprender. Así, la primera causa de emprendimiento está relacionada con el entorno; en la época actual, el mercado laboral no es capaz de absorber la demanda de empleo. Otros motivos alegados son la autorrealización personal o el hecho de disponer de la experiencia laboral previa necesaria.

\footnotetext{
Madrid.

${ }^{8}$ La información referida al emprendimiento individual, se completa con el proyecto de investigación titulado "El emprendimiento individual (trabajo autónomo) como vía de acceso al mercado laboral por parte de los estudiantes y graduados universitarios" financiado por el Ministerio de Trabajo y Seguridad Social.
}

REVESCO No 112 - Extraordinario en Homenaje al Profesor Alfonso Carlos MORALES GUTIÉRREZ - ISSN: 1885-8031 - www.ucm.es/info/revesco 
En segundo lugar, si de las formas empresariales participativas mencionadas más arriba, el trabajo autónomo es la opción elegida por los jóvenes emprendedores, cabe preguntarse cuáles son los motivos por los cuales la eligen; y por el contrario, cuales son las que procuran que los jóvenes no sean proclives a elegir las fórmulas participativas de sociedades laborales y sociedades cooperativas de trabajo asociado.

\subsection{Valoración de la elección del revestimiento jurídico por parte de los jóvenes emprendedores.}

\subsubsection{El trabajo autónomo.}

La principal razón de la elección del trabajo autónomo como fórmula empresarial se relaciona con el tamaño; cuando los nuevos emprendedores tienen en mente la puesta en marcha de proyectos empresariales, piensan que la llevaran a cabo ellos mismos, individualmente, o como máximo dos personas, lo que limita la adopción de otras formas jurídicas por razón del número de promotores ${ }^{9}$.

Los motivos que les inducen a la elección de la forma jurídica (ALONSO y GALVE: 2008) aparecen recogidos en la siguiente tabla. En ella se evidencia que en el 71,43 por ciento de los casos la selección del trabajo autónomo se basa en el hecho de querer ser un profesional independiente, así como por tratarse de la fórmula empresarial menos costosa para iniciar la actividad. El 57,14 por ciento de los encuestados también valoran la libertad de establecerse por cuenta propia a la hora en su elección mientras que en el 42,86 por ciento de los casos analizados ponen en valor la sencilla tramitación, la flexibilidad y al independencia. Sólo en el 28,57 por ciento de los casos su elección se basa en el conocimiento en su entorno de trabajadores autónomos con experiencias positivas, en el hecho de no ver limitada la carrera profesional por bloqueos en el ascenso laboral o por tener derecho a percibir la prestación por desempleo en pago único, o por tener acceso a subvenciones.

\footnotetext{
${ }^{9}$ Esta situación no se produce en el caso de las Empresas de Base Tecnológica cuyo número de promotores es de tres o cuatro personas.
} 
Tabla 4 - Motivos por los que los emprendedores eligen la forma jurídica

\begin{tabular}{|l|c|}
\hline Motivos que inducen al emprendedor a la elección de la forma jurídica & $\begin{array}{l}\text { Porcentaje de } \\
\text { emprendedores que lo } \\
\text { han seleccionado }\end{array}$ \\
\hline $\begin{array}{l}\text { Quiero ser un profesional independiente } \\
\text { Es una fórmula empresarial menos costosa para iniciar la actividad }\end{array}$ & 71,43 \\
\hline Valoro la libertad que tiene establecerse por cuenta propia & 57,14 \\
\hline $\begin{array}{l}\text { Los trámites son sencillos } \\
\text { Es una fórmula empresarial flexible para empezar la actividad } \\
\text { Quiero ser mi propio jefe }\end{array}$ & 42,86 \\
\hline $\begin{array}{l}\text { Conozco en mi entorno trabajadores autónomos y su experiencia es buena } \\
\text { Para no ver limitada mi carrera profesional por bloqueos en el ascenso } \\
\text { laboral } \\
\text { Tengo / tenemos derecho a percibir la prestación por desempleo en pago } \\
\text { único } \\
\text { El acceso a subvenciones }\end{array}$ \\
\hline $\begin{array}{l}\text { En la época actual el mercado laboral no a la demanda de empleo } \\
\text { La fiscalidad favorece } \\
\text { Por motivos de autorrealización personal } \\
\text { Porque dispongo de la experiencia laboral previa necesaria } \\
\text { Los principios y valores por los que rigen su funcionamiento }\end{array}$ \\
\hline $\begin{array}{l}\text { Por considerar que puedo obtener mayor nivel de ingresos } \\
\text { Somos tres socios trabajadores } \\
\text { Somos dos socios trabajadores y, al menos, un socio capitalista } \\
\text { La primacía de las personas sobre el capital social } \\
\text { La limitación de la responsabilidad }\end{array}$ \\
\hline
\end{tabular}

Fuente: Elaboración propia

\subsubsection{Las sociedades laborales y las sociedades cooperativas de trabajo asociado.}

Los jóvenes emprendedores no se platean, en general, otras formas jurídicas participativas alternativas como son las Sociedades Limitadas Laborales, o las Sociedades Cooperativas de Trabajo Asociado.

No contemplar estas posibilidades es derivado de varios motivos; el primero, un obstáculo insalvable, la limitación del número mínimo de promotores que no es acorde con el número mínimo de socios exigidos por estas fórmulas jurídicas; el segundo, el desconocimiento generalizado de estas fórmulas jurídicas, no sólo de los promotores sino también de los asesores y prescriptores que los acompañan.

Dicho desconocimiento les hace mostrar una actitud defensiva, en lugar de receptiva, hacia la información que se les pueda ofrecer sobre las mismas. 
Así, los nuevos emprendedores procedentes de la comunidad universitaria son más proclives a la utilización de las formas jurídicas objeto del presente trabajo cuando disponen de información sobre las mismas, las conocen y reconocen los valores que están inmersos en las empresas de participación.

En este caso, es destacable la importancia de que en los niveles formativos superiores, y también en los de grado medio, se incorporen materia relacionadas con las empresas de participación, de modo, que en el ámbito de las ciencias sociales, que es en el que los emprendedores reciben una mayor información sobre las mismas es en el que estas fórmula jurídicas son consideradas en mayor medida.

Por otra parte, es preciso tener en cuenta que si el negocio tiene éxito y prospera, consideran revestirse jurídicamente bajo forma societaria buscando una consolidación en el mercado, aunque principalmente sea como Sociedad de Responsabilidad Limitada.

\subsection{Valoración de los problemas planteados para la elección de la forma jurídica.}

La siguiente tabla trata de comparar los problemas e inconvenientes considerados por los jóvenes emprendedores con respecto al grado de información de que disponen cuando deciden acometer un proyecto empresarial.

La comparativa es el resultado de la valoración de un conjunto de problemas de 1 a 5 (1 no supone problema, 5 problema de gran envergadura) sobre los que han sido consultados. 
Gráfico 1- Comparativa de los problemas destacados para el emprendimiento según base jurídica

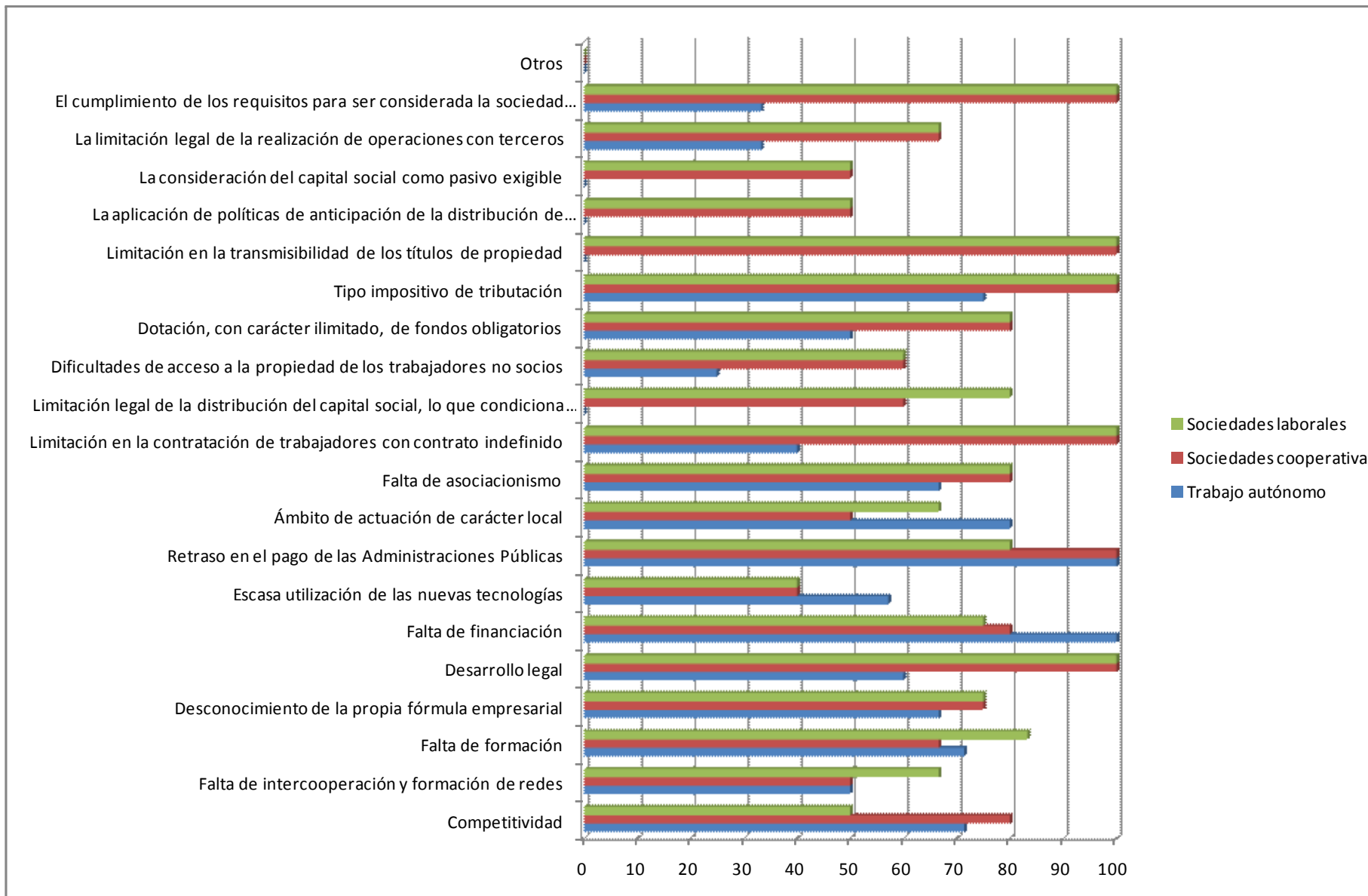

Fuente: Elaboración propia.

Excepto en casos muy concretos, los jóvenes otorgan el mismo grado de importancia a los problemas en el caso de las sociedades laborales y las sociedades cooperativas de trabajo asociado diferenciándolos de los problemas con los que se enfrenta el trabajador autónomo.

El desarrollo legal, las limitaciones en la contratación de trabajadores con contrato indefinido, la fiscalidad y las limitaciones para la transmisibilidad de las aportaciones en el caso de las sociedades cooperativas y las laborales constituyen un primer bloque de problemas considerados importantes, muy importantes o de gran envergadura para las sociedades cooperativas y laborales.

Un segundo bloque de problemas a los que los entrevistados otorgan importancia en el caso de las sociedades cooperativas y sociedades laborales alejándose de los que consideran importantes en el caso de los trabajadores autónomos se refieren a: las limitaciones para realizar operaciones con terceros, la obligatoriedad de dotar fondos de reserva, las REVESCO Nº 112 - Extraordinario en Homenaje al Profesor Alfonso Carlos MORALES GUTIÉRREZ - ISSN: 1885-8031 - www.ucm.es/info/revesco 
limitaciones en la distribución del capital social que condiciona las dificultades de acceso a los trabajadores no socios.

Un tercer bloque lo constituyen aquellos problemas considerados importantes tanto para las sociedades cooperativas y laborales como para los trabajadores autónomos. Estos son: la falta de asociacionismo, la falta de financiación, el desconocimiento de la propia fórmula empresarial, la formación y la competitividad.

El último bloque lo constituyen problemas aislados que sólo son tenidos en cuenta como importantes, o muy importantes, para el caso del trabajo autónomo; a saber, el ámbito local que limita su actividad y el escaso uso de las nuevas tecnologías.

De los resultados obtenidos se pone en evidencia la falta de conocimiento de las sociedades laborales y las sociedades cooperativas de trabajo asociado por parte de los nuevos emprendedores universitarios dado que problemas como la falta de intercooperación y formación de redes se consideran irrelevantes (MARTíN: 2010). También se pone de manifiesto en el caso del trabajo autónomo. Si esta apreciación sorprendía en las formas jurídicas analizadas previamente, sorprende en mayor medida en el caso del trabajador autónomo, dado que la intercooperación, y el hecho de formar parte de una red permiten suplir las deficiencias individuales de sus miembros, sin que recaiga en una única persona la necesidad de contar con las cualidades básicas, la experiencia necesaria, y los conocimientos mínimos para que la empresa salga adelante (GARCÍA-GUTIÉRREZ, LEJARRIAGA, FERNÁNDEZ y MARTÍN: 2006; DÍAZ, GARCÍA, RUIZ, JAIMEZ y HERNÁNDEZ: 2009, p. 8).

De todo lo expuesto anteriormente, se pone en evidencia la falta de conocimiento por parte de los nuevos emprendedores universitarios de las empresas de participación, así como, la insuficiente formación por parte de los técnicos de estructuras de apoyo sobre estas formas jurídicas. Es por ello, que ante esta situación se requiere la implicación efectiva del profesorado que investiga en materia de emprendedores, en general, y de empresas de economía social, en particular, con las estructuras de apoyo para favorecer la concreción de nuevas iniciativas emprendedoras bajo fórmulas como el trabajo autónomo, las Sociedades Laborales y las Sociedades Cooperativas de Trabajo Asociado. 
A este respecto, el 57,14 por ciento de los emprendedores encuestados no conoce si en su universidad hay alguna materia específica que estudie la economía social, mientras que dicho porcentaje se eleva al 85,71 por ciento en cuanto al desconocimiento de si existe alguna materia específica que estudie el trabajo autónomo; no obstante, el 71,43 por ciento de los encuestados valoran de forma positiva su inclusión en los planes de estudios (en muchos casos se habrá perdido una buena oportunidad para ello en la elaboración de los nuevos estudios de Grado) lo que pone de manifiesto la valoración positiva que realizan sobre la consideración de estas materias en sus planes de estudios (BENAVIDES, SÁNCHEZ y LUNA: 2004).

\section{EL EMPRENDIMIENTO EN EL ÁMBITO UNIVERSITARIO: LA EXPERIENCIA DE LA UNIVERSIDAD COMLUTENSE DE MADRID}

Entre las funciones más importantes de la Universidad se encuentra la preparación de los discentes para el ejercicio de actividades profesionales mediante la capacitación oportuna para ello. En este sentido, la Universidad Complutense de Madrid se ha sumado a la iniciativa de otras universidades españolas y de más allá de nuestras fronteras consistente en proveer de una estructura apropiada para apoyar las propuestas de actividades emprendedoras que pudieran formular los integrantes de la comunidad universitaria.

Así surge la Oficina Complutense del Emprendedor (COMPLUEMPRENDE), que se plantea como un espacio de relación para todos aquellos agentes de la Universidad Complutense que puedan estar implicados, de una u otra forma, con el emprendimiento y su cultura, sirviendo de apoyo a quienes estén interesados en la creación de empresas.

COMPLUEMPRENDE tiene su origen en el Decreto Rectoral 1/2007 de 2 de enero. $\mathrm{Su}$ misión fundamental es la difusión de la cultura emprendedora y el apoyo a lo emprendedores. En el orden práctico la oficina de COMPLUEMPRENDE no comienza su actividad operativa hasta el mes de julio de 2007, pero desde que empezara su andadura hasta la actualidad ha ido incrementando progresivamente su actividad. Prueba de ello es el incremento experimentado tanto en el número de personas atendidas, como en la firma de convenios de colaboración con distintas entidades, y la apertura de nuevo punto de asesoramiento en el año 2009 en el Campus de Ciudad Universitaria.

REVESCO No 112 - Extraordinario en Homenaje al Profesor Alfonso Carlos MORALES

GUTIÉRREZ - ISSN: 1885-8031 - www.ucm.es/info/revesco 
El servicio prestado por la Oficina Complutense del Emprendedor está dirigido a los miembros de la Universidad Complutense, tanto al personal docente e investigador, como al personal de administración y servicios, estudiantes, licenciados y diplomados.

Desde COMPLUEMPREDE se busca el fomento de la vocación emprendedora entre los miembros de la comunidad universitaria (mediante la realización de sesiones de difusión, sensibilización y reuniones personales), el apoyo y orientación a los emprendedores (mediante la elaboración de un plan de negocio), y la formación de los futuros emprendedores para mejorar sus niveles y conocimientos empresariales.

Para llevar a cabo las actividades anteriormente descritas se pone a disposición de los emprendedores recursos informativos sobre creación de empresas, un espacio para recibir asesoramiento sobre la forma de crear una empresa, y un entorno donde adquirir y mejorar los conocimientos sobre creación de empresas y capacidades empresariales (KUSHELL: 2002).

Cabe destacar, en el caso de las labores de asesoramiento, que en COMPLUEMPRENDE se realizan reuniones personales orientadas a ayudar al emprendedor en la creación de una empresa y en la elaboración del correspondiente plan de negocio, reuniones personales tutorizadas en las que se simula la creación de una empresa y se elabora el plan de negocio dirigidas a estudiantes (y con las que se pueden conseguir un crédito de libre configuración), asesoramiento de proyectos premiados en el Campus del Emprendedor, asesoramiento de proyectos que participan en la Competición Nacional de Emprendedores Universitarios Graduate Programme (Fundación Universidad Empresa y Fundación Junior Achievement), así como asesoramiento a investigadores para la presentación ante el Vicerrectorado de Investigación de propuestas viables para la creación de empresas de base tecnológica.

Entre los años 2007 y 2012, el número de emprendedores que han accedido a los servicios prestados por COMPLUEMPRENDE ascendió a 1.274, con una distribución similar por sexos (55,4 por ciento mujeres) y mayoritariamente en la franja de edad entre 18 y 25 años (51 por ciento).

La segunda actividad principal que se lleva a cabo en COMPLUEMPRENDE es la labor de formación, cuyo objetivo es mejorar el nivel de conocimientos y capacidades 
empresariales con acciones formativas, así como fomentar el espíritu emprendedor entre los estudiantes universitarios. Para ello se realizan:

- Entrevistas de asesoramiento para la creación de empresas de carácter individual durante tres sesiones de dos horas de duración cada una, y una cuarta para la elaboración del plan de empresa. Dicha actividad lleva asociado el reconocimiento de un crédito de libre elección.

- Formación y orientación para la creación de empresas durante tres sesiones de dos horas de duración cada una, y una cuarta para la elaboración de un plan de empresa, y la asistencia a un taller de información y motivación para la creación de empresas de 4 horas de duración. Dicha actividad lleva asociado el reconocimiento de un crédito y medio de libre elección.

- Laboratorio empresarial que consta de cuatro sesiones de 2,5 horas de duración cada una de ellas, siendo una acción colectiva con la que se pretende crear y desarrollar una empresa en equipo. Dicha actividad lleva asociado el reconocimiento de un crédito de libre elección.

- Taller de habilidades empresariales con una duración de 6 sesiones de 4 horas cada una de ellas, y con un reconocimiento de 2 créditos y medio de libre elección.

- Taller de creación de empresas con una duración de 7 sesiones de 4 horas cada una de ellas, y con un reconocimiento de 3 créditos de libre elección.

Desde COMPLUEMPRENDE se ha intentado en todo momento orientar a los potenciales emprendedores universitarios hacia la economía social o empresas de participación.

Un ejemplo de ello es el Encuentro de Verano "Universidad y Emprendizaje", que tuvo lugar los días 16, 17 y 18 de julio, celebrado en el marco de los Cursos de Verano del Escorial 2008 y organizado por COMPLUEMPRENDE, con el patrocinio de la Agencia de Desarrollo "Madrid Emprende" del Ayuntamiento de Madrid, y la colaboración de la Escuela de Estudios Cooperativos, y la Cátedra de Empresa Individual y Trabajo Autónomo de la Universidad Complutense de Madrid. 
Con el encuentro se pretendía, entre otros objetivos, presentar la situación actual de los colectivos con dificultades de inserción laboral, así como las distintas fórmulas desarrolladas por distintas organizaciones para mejorar y fomentar el emprendizaje en su entorno. Desde proyectos de la iniciativa comunitaria EQUAL, como es el caso del CRIO (Creación de Empresas e Igualdad de Oportunidades), se evidencia el destacado papel que el Trabajo Autónomo, Sociedades Cooperativas y Sociedades Laborales desempeñan en la inserción laboral de los colectivos referidos.

Una de las principales actuaciones de COMPLUEMPRENDE tiene que ver con su participación en RedEmprendia, red integrada por 20 Universidades iberoamericanas que cuenta con el apoyo de Universia y el Banco de Santander que tiene como objetivo estratégico fundamental "Trasladar la apuesta por una "Universidad Emprendedora" a todos los países de Iberoamérica y a sus universidades, ayudando a crear una fuerte cultura de la innovación y el emprendimiento en las comunidades universitarias, que se traslade también a la sociedad y que sea institucionalizada como una nueva e imprescindible misión, al igual que lo es la docencia y la investigación" (http://www.redemprendia.org/quienes-somos/mision-yvision).

Son muy diversos los programas que se desarrollan en la red, entre los que cabe destacar:

1. Programa de hospedaje : El objetivo del programa es apoyar la internacionalización de pequeñas y medianas empresas surgidas de las universidades, así como a emprendedores, técnicos y profesionales del emprendimiento en la mejora de sus conocimientos y capacidades, financiándoles estancias de entre 2 y 12 meses en alguno de los nodos de la red. Está organizándolo el Parque Científico de Madrid y ya hay un número considerable de interesados en el intercambio.

2. Directorio de empresas: Se está elaborando un directorio en la Web de RedEmprendia de las empresas localizadas en incubadoras, aceleradoras y parques científicos y/o tecnológicos de la Red, con el objetivo de otorgar una exposición internacional a las mismas para ampliar la posibilidad de acciones sinérgicas o complementarias entre ellas. 
3. Indicadores de transferencia, innovación y emprendimiento: El proyecto trata de identificar, estandarizar y aplicar un conjunto de indicadores de Innovación y Emprendimiento en las quince universidades de la Red. Una vez definido este conjunto de indicadores se dispondrá de un sistema de monitorización en el tiempo sobre la actividad en este ámbito de las Universidades socias. Se prevé realizar una publicación con los datos derivados de la primera encuesta de indicadores

4. Instituto de Emprendimiento ( $\mathrm{IE}^{2}$ ): Con esta actividad se persigue difundir la oferta y los recursos de formación de las universidades miembros, así como organizar nuevas ofertas formativas y otro tipo de productos y actividades para la puesta en común de conocimiento propio del ámbito de la innovación y el emprendimiento.

5. Concurso de ideas, proyectos empresariales e iniciativas emprendedoras: Con la convocatoria del concurso de pretende premiar a las mejores ideas, proyectos empresariales (sin constituir) y mejores iniciativas spinoff de las universidades de la red, seleccionándolas de entre los mejores de los concursos individuales (anuales) de cada una de ellas

6. Programa de sabáticos a académicos emprendedores: Programa orientado a la identificación, apoyo y reconocimiento a emprendedores miembros de las comunidades universitarias de la Red, para que puedan dedicarse a tiempo completo, en períodos de un semestre a un año, a la puesta en marcha o consolidación de proyectos de emprendimiento.

7. Programa de visitas a nodos: Mediante esta acción se está llevando a cabo la organización y promoción de visitas a la Red y a sus nodos por parte de otras universidades - preferentemente iberoamericanas, pero también de otros países-, organismos, instituciones, etc. Estas visitas comprenden tanto visitas físicas, como formación que se ofrezca, bien sea como parte de una visita, o de forma independiente, a aquellas personas interesadas en ello.

8. Difusión de instrumentos y buenas prácticas: Se ha realizado una primera publicación que recopila algunas de las iniciativas más singulares de apoyo al emprendimiento que han sido o están siendo desarrolladas y aplicadas en las universidades que forman parte de RedEmprendia. El objetivo es generar un documento -impreso y en línea- de 
consulta para la búsqueda de modelos e iniciativas que puedan ser de utilidad para futuros desarrollos en las universidades, tanto de la Red como externas a la misma

9. Casos de éxito de emprendedores. Libro de casos: Con este proyecto se propone realizar un análisis de casos de éxito entre las empresas incubadas, de forma que se pueda generar una ficha para cada una de ellas que pueda ser incluida en la página web.

10. Movilidad para jóvenes emprendedores: Con el desarrollo de este proyecto RedEmprendia trata de promover el intercambio de experiencias, el aprendizaje intercultural, la formación y la generación de contactos internacionales entre jóvenes emprendedores que han pensado transformar en empresa su proyecto empresarial, a través de la realización de estancias formativas en empresas vinculadas a los miembros de RedEmprendia, con una duración de entre 2 y 6 meses.

Este último es el asunto más importante, a nuestro entender.

El principal objetivo del Programa es ayudar a nuevos emprendedores y empresarios a desarrollar actividades emprendedoras basadas en el conocimiento y el desarrollo tecnológico, en la adquisición de experiencia y habilidades en la gestión de empresas preferentemente en entidades de otro país.

El programa subvenciona los gastos de la estancia con un mínimo de 3.000€ (para una estancia de 2 meses) y un máximo de $7.000 €$ (para una estancia de 6 meses), y está dirigido a nuevos emprendedores de los colectivos identificados por cada Universidad participante (Ejemplo: alumnos de máster, doctorandos, doctores en los primeros años tras la consecución del título de doctor, estudiantes de grado, graduados en los últimos años, emprendedores vinculados a la Universidad con empresas creadas hace menos de un año, etc.). En todo caso el nuevo emprendedor deberá ser mayor de edad y contar con un proyecto empresarial o una idea de negocio, que se presentará a través de un plan de negocio completo, además de poseer la formación adecuada para llevarlo a cabo.

La empresas receptoras son entidades interesadas en ofrecer estancias a los nuevos emprendedores. Se trata de empresas -privadas, públicas, asociaciones u otras formas jurídicas- consolidadas en su sector y con un mínimo de 3 años de actividad. 
Esta experiencia puede trasladarse a otras Redes especializadas en colectivos concretos, como es el caso de RULESCOOP, lo que permitiría potenciar la creación de nuevas iniciativas emprendedoras en forma de sociedades cooperativas o de cualquier otra entidad con forma de organización de participación o de economía social.

\section{CONCLUSIONES}

En España existe un importante problema de desempleo, que a raíz de la actual crisis económica se ha agravado de forma considerable durante los últimos años, consecuencia de la reducción del número de empresas que se crean, y del incremento del cierre, y las reducciones de plantilla, de las ya existentes. Los jóvenes son uno de los colectivos más afectados por el desempleo, la temporalidad de los contratos, y las jornadas a tiempo parcial, por lo que, ante la dificultad de encontrar empleo por cuenta ajena, el emprendimiento, y en concreto el emprendimiento colectivo como empresas de trabajo asociado, puede suponer una alternativa al trabajo asalariado que les permita tanto incorporarse, como mantenerse en el mercado laboral, y mejorar sus condiciones de trabajo.

No cabe duda de que el emprendimiento constituye la base del crecimiento económico, y es ahí donde las empresas de participación de trabajo asociado pueden desempeñar un papel clave en la recuperación económica de nuestro país. Durante los últimos años la tendencia ha sido hacia la reducción en el ritmo de creación de estas entidades, habiendo tenido lugar un cierto estancamiento en la generación de empleo de carácter participativo. No obstante, los últimos datos del año 2010 son más positivos que los de los años previos, en especial en el caso de las sociedades cooperativas de trabajo asociado, por lo que invitan a pensar en el optimismo y en la recuperación de la senda de crecimiento. Además se trata de empresas que contribuyen al desarrollo territorial, en las que es inherente a su condición una especial sensibilidad en términos de responsabilidad social, que no se deslocalizan y que permiten así una mayor integración y cohesión social aportando al mismo tiempo riqueza y empleo de calidad y estable allí dónde se encuentran. Es por ello que también son fórmulas adecuadas para dar respuesta a los retos del cambio demográfico, como pueden ser el relevo generacional, la igualdad efectiva de la mujer, o la incorporación de la población inmigrante al mercado laboral. Pero su importancia y sus bondades no sólo son reconocidas a nivel nacional, sino que estas empresas son consideradas desde la Unión Europea como modelos a seguir para la implantación y difusión de los planes de participación de las empresas 
independientemente de su tamaño (en la mayor parte de los casos se trata de microempresas, e incluso "nanoempresas"), dados los beneficios que ello conlleva tanto para las propias empresas, como para los socios trabajadores, y para la zona en la que se localizan.

Hay que tener en cuenta que el nacimiento de las empresas de participación de trabajo asociado está vinculado a la presencia de un número mínimo de tres socios, siendo la alternativa adecuada para los casos de emprendimiento colectivo, y tratándose en la mayoría de los casos de microempresas y pequeñas o medianas empresas. Por tanto, la dimensión inicial supone un freno en la elección de las formas jurídicas de trabajo asociado por parte de los emprendedores universitarios dado que en la mayor parte de los casos se trata de un único promotor, o a lo sumo dos, por lo que para cumplir el requisito legal requerirían de la presencia de al menos un socio capitalista o colaborador. Esta limitación legal ha sido una de las principales causas por las que muchas de estas empresas han sido descalificadas y se han transformado en otras formas jurídicas para poder continuar desarrollando su actividad empresarial, aun conservando su carácter participativo.

Es habitual que los nuevos emprendedores tengan en mente la puesta en marcha de proyectos empresariales de forma unipersonal. No obstante, las empresas de participación de trabajo asociado también han de constituir el vehículo adecuado para que las iniciativas empresariales llevadas a cabo por trabajadores autónomos, o empresarios individuales, se concreten bien en sociedades laborales, o en sociedades cooperativas de trabajo asociado, cuando necesiten aumentar su dimensión y requieran de formas jurídicas de carácter societario para afianzar sus negocios.

El hecho de que no exista una demanda efectiva de información sobre las fórmulas de trabajo asociado entre los jóvenes universitarios pone de manifiesto el desconocimiento de su existencia. Esto unido a los problemas de imagen que existen en la sociedad justifica el hecho de que haya más empresas de participación de trabajo asociado de hecho que de derecho. Así, existe una estrecha relación entre la imagen que se tiene de estas sociedades, y el grado de conocimiento de las mismas, en el sentido de que a mayor nivel de desconocimiento la imagen que se tiene de estas sociedades es más negativa, lo que hace que los emprendedores adopten una actitud defensiva, y prefieran optar para la puesta en marcha de sus proyectos empresariales por otras formas jurídicas consideradas convencionales. Es por ello que se considera de vital importancia tanto la labor de asesoramiento de los técnicos de las 
estructuras de apoyo creadas en el ámbito universitario, como el hecho de que éstos puedan contar con la colaboración del personal docente que investiga en materia de emprendedores y de empresas de trabajo asociado, con el objetivo de poder favorecer la concreción de nuevos proyectos empresariales bajo estas formas jurídicas. No obstante, desde las organizaciones representativas también se debe llevar a cabo una labor de difusión de estas formas jurídicas como formas naturales de emprender.

Desde la Oficina de Compluemprende de la Universidad Complutense de Madrid se trabaja en esta línea, promoviendo y difundiendo la cultura emprendedora y el apoyo a los emprendedores entre los miembros de la comunidad universitaria, prestando para ello asesoramiento y formación. Desde esta oficina se ha intentado en todo momento orientar a los potenciales emprendedores universitarios hacia la economía social o empresas de participación. Son múltiples las actividades llevadas a cabo desde Compluemprende para lograr estos objetivos, siendo destacable su participación en la RedEmprendia, cuya experiencia podría trasladarse a redes especializadas como es el caso de RULESCOOP para potenciar la creación de sociedades cooperativas o de cualquier otra entidad con forma de organización de participación o de economía social.

\section{BIBLIOGRAFÍA}

ALONSO NUEZ, M ${ }^{a}$ J; GAVE GÓRRIZ, C (2008). El emprendedor y la empresa: una revisión teórica de los determinantes a su constitución. Acciones e Investigaciones Sociales, $\mathrm{n}^{\circ} 26$, julio, p. 5-44.

ATA. Federación Autónomos. Informe jóvenes emprendedores - Unión Europea, 2011. Disponible en en: http://www.ata.es/imagenes/publicacionesPdf/publicacion55.pdf

BEL DURÁN, P (2010). El trabajo autónomo desde la perspectiva del estudiante universitario. Una aproximación desde los estudios de ADE. Ponencia presentada al I Congreso de la Red Universitaria de Trabajo Autónomo (RUTA), Santiago de Compostela, octubre.

BEN-NER, A (1988). The life cycle of worker-owned firms in market economies. A theoretical analysis. Journal of Economic Behaviour \& Organization, vol. 10, $\mathrm{n}^{\mathrm{o}} 3$, octubre, p. 287-313. 
BENAVIDES ESPINOSA, Mª M; SÁNCHEZ GARCÍA, Mª I; LUNA AROCAS, R (2004).

El proceso de aprendizaje para los emprendedores en la situación actual: un análisis cualitativo en el ámbito universitario. Dirección y Organización, no 30, abril, p. 34-48.

CLEMENTE LÓPEZ, J; DÍAZ FONSECA, M y MARCUELLO SERVÓS, C. (2009) Sociedades Cooperativas y Sociedades laborales en España: Estudio de su contribución a la creación de empleo y al crecimiento económico. REVESCO. Revista de Estudios Cooperativos, $\mathrm{n}^{\circ}$ 98, segundo cuatrimestre, pp. 35-69.

COMITÉ ECONÓMICO Y SOCIAL EUROPEO. Dictamen SOC/371 sobre participación financiera de los trabajadores en Europa. Disponible en internet en: http://www.eesc.europa.eu

DÍAZ BRETONES, F (2004). Actitudes, motivación y creación de empresas: el caso de los emprendedores en sistema cooperativo. Revista de Estudios Cooperativos (REVESCO), $\mathrm{n}^{\circ}$ 84 , tercer cuatrimestre, p. 37-52.

DÍAZ BRETONES, F; GARCÍA MARTÍ, E; RUIZ JIMÉNEZ, C; JAIMEZ ROMÁN, M y HERNÁNDEZ ORTIZ, M $\mathbf{M}^{\mathrm{a}} \mathrm{J}$ (2009). Actitudes, valores y comportamientos emprendedores en la población universitaria. Ponencia presentada al II Congreso Internacional y $\mathrm{X}$ Seminario Iberoamericano Motiva: La Universidad y El Emprendimiento, celebrado entre el 27 y el 30 de octubre en Valencia.

DICKSTEIN, C (1991). The Promise and Problems of Worker Cooperatives. Journal of Planning Literature, $\mathrm{n}^{\circ}$ 6, p. 16-33

ESPAÑA. LEY 4/1997, de 24 de marzo, de Sociedades Laborales. BOE $n^{\circ} 72$, de 25 de marzo.

ESPAÑA. LEY 27/1999, de 16 de julio, de Cooperativas. BOE nº 170, de 17 de julio. FERNÁNDEZ GUADAÑO, J.; y MARTÍN LÓPEZ, S. Y LEJARRIAGA PÉREZ de las VACAS, G (2010). "Retos del trabajo autónomo para afrontar la crisis económicofinanciera”. Economistas. Revista del Colegio de Economistas, n 124, pp. 99-113.

GARCÍA-GUTIÉRREZ FERNÁNDEZ, C (2002). "La empresa de participación: características que la definen. Virtualidad y perspectivas en la sociedad de la información". CIRIEC-España: Revista de economía pública, social y cooperativa, $\mathrm{n}^{\circ} 40$, abril, p. 99 122.

GARCÍA-GUTIÉRREZ FERNÁNDEZ, C y LEJARRIAGA PÉREZ DE LAS VACAS, G. (Directores) (2004). Libro Blanco de las empresas de participación de trabajo (economía social) de la Ciudad de Madrid. Madrid: Madrid Emprende. 
GARCÍA-GUTIÉRREZ FERNÁNDEZ, C; LEJARRIAGA PÉREZ DE LAS VACAS, G; BEL DURÁN, P; FERNÁNDEZ GUADAÑO, J y MARTÍN LÓPEZ, S (2008). El emprendimiento y el empleo a través de la empresa individual: contraste de las fuentes estadísticas. REVESCO. Revista de Estudios Cooperativos, $\mathrm{n}^{\circ}$ 96, p. 16-48.

GARCÍA-GUTIÉRREZ FERNÁNDEZ, C; LEJARRIAGA PÉREZ DE LAS VACAS, G; FERNÁNDEZ GUADAÑO, J y MARTÍN LÓPEZ, S (2006). El trabajo autónomo desde la perspectiva de la política empresarial. En: SALINAS RAMOS, F y HERRANZ DE LA CASA, J. Ma. (Editores). El Trabajo Autónomo en España. Salamanca: Universidad Católica de Ávila, p. 97-127.

GRÁVALOS, M.A.; POMARES, I (2001): “La adaptación de las Sociedades Laborales a la evolución del ciclo económico. Un estudio empírico para las diferentes comunidades autónomas". CIRIEC-España, Revista Pública, Social y Cooperativa, , no 38, pp. 33-55.

INSTITUTO NACIONAL DE ESTADÍSTICA (INE). Disponible en Internet en: www.ine.es KUSHELL, J (2002). Solo para emprendedores. Grupo editorial Norma.

LEJARRIAGA, G y MARTÍN, S. (2010): “Las sociedades laborales”. En: MONZÓN, J. L (Director) (2010). La Economía Social en España en el año 2008. CIRIEC-España, Valencia.

LEJARRIAGA PÉREZ DE LAS VACAS; MARTÍN LÓPEZ, S (2010). El nacimiento de nuevas iniciativas emprendedoras como empresas de participación en el ámbito universitario: el apoyo prestado desde Compluemprende. Ponencia presentada a las XIII Jornadas de Investigadores, CIRIEC-España, Zaragoza, octubre.

MARTÍN LÓPEZ, S (2010). La necesidad de reactivación del crecimiento de las sociedades laborales y la reforma de su legislación: Análisis económico-financiero de sus principales implicaciones. REVESCO. Revista de Estudios Cooperativos, $\mathrm{n}^{\circ} 102,2^{\circ}$ Cuatrimestre.

MARTÍN LÓPEZ, S; LEJARRIAGA PÉREZ DE LAS VACAS, G e ITURRIOZ DEL CAMPO, J (2007). Valoración de la adaptación de las empresas de participación de trabajo asociado en España a las modalidades contempladas en los informes PEPPER. Ponencia presentada al primer Congreso Internacional de CIRIEC "Strengthening and Building Communities: The Social Economy in a Changing World”. Victoria (Canadá), 22-25 octubre.

MELGAREJO MOLINA, Z; ARCELUS, F.J y SIMÓN, K (2007). Una evaluación crítica del potencial de supervivencia de las sociedades laborales. CIRIEC-España, Revista de Economía Pública, Social y Cooperativa, nº 59, octubre, pp. 181-202.

REVESCO No 112 - Extraordinario en Homenaje al Profesor Alfonso Carlos MORALES GUTIÉRREZ - ISSN: 1885-8031 - www.ucm.es/info/revesco 
MINISTERIO DE TRABAJO E INMIGRACIÓN. Disponible en Internet en: www.mtin.es

MONTOLIO HERNÁNDEZ, J. M M $^{\mathrm{a}}$ (2002). Economía social: concepto, contenido y significación en España. CIRIEC-España, Revista de Economía Pública, Social y Cooperativa, $\mathrm{n}^{\mathrm{o}}$ 42, noviembre, pp. 5-31.

MONZÓN CAMPOS, J. L (Director) (2010a). Economía Social y su impacto en la generación de empleo. Madrid: FUNDIBES.

MONZÓN CAMPOS, J. L (Director) (2010b). La Economía Social en España en el año 2008. Valencia: CIRIEC-Esapaña.

MORALES GUTIÉRREZ, A.C; MARTÍN LÓPEZ, S and LEJARRIAGA PÉREZ DE LAS VACAS, G (2008). Labour Managed Firms in Spain. CIRIEC- España, Revista de Economía Pública, Social y Cooperativa, nº 62, Special Issue, October, pp. 61 - 85.

TOMAS, J.A. (1995): “Transformación económica, Economía Social y Empleo”. Noticias de la Economía Pública, Social y Cooperativa, no . 15, pp. 25 - 31. 